\title{
Caracterización y actitudes de los horticultores de la Región Metropolitana de Santiago, Chile
}

\author{
Characterization and attitudes of vegetable growers in the Santiago Metropolitan \\ Region, Chile
}

Sofía Boza ${ }^{1 *}$, Maruja Cortés ${ }^{1}$, Carmen Prieto ${ }^{2}$, Tomás Muñoz ${ }^{1}$

\begin{abstract}
RESUMEN
La producción de hortalizas es fundamental para sostener la oferta nacional de productos frescos. El principal polo de producción en Chile es la Región Metropolitana de Santiago (RMS). El objetivo de la presente investigación es caracterizar a los productores hortícolas de la RMS, así como determinar sus actitudes respecto a su actividad. Para ello se encuestó de forma presencial a un total de 170 productores distribuidos en 12 comunas de la región. Los datos obtenidos fueron tratados por medio de técnicas descriptivas. Entre los resultados alcanzados destacan los siguientes: I) el promedio de edad es de 54,14 años, II) el 54,5\% de los encuestados no tiene educación formal o ha finalizado solo la básica, III) hay un buen acceso a servicios y movilización, IV) el promedio de hectáreas productivas es 7,68, pero con alta dispersión, V) el uso de invernadero, técnicas de agricultura orgánica e hidroponía es muy limitado, así como la contratación de mano de obra permanente, VI) el uso de las Tecnologías de la Información y Comunicación (TIC) - excepto celular - es escaso, VII) la innovación declarada se centra en aspectos técnico-productivos, VIII) la mayor parte de los encuestados no se asocia; su relación con el sector público es acotada, vende a través de intermediarios y no lleva registro de sus operaciones. Respecto a sus actitudes, destaca la valoración positiva de la manera en la cual realizan su actividad productiva; sin embargo, existe incertidumbre de cara al futuro de esta. A partir de lo anterior es posible concluir que es importante llevar a cabo acciones que deriven en una mejora no solo de los aspectos técnico-productivos, sino también de aquellos relacionados con la gestión, la comercialización y el acceso a TIC. Lo anterior debe ir acompañado de un intercambio con los productores que les permita convencerse de las oportunidades de incorporar ciertas modificaciones en su quehacer.
\end{abstract}

Palabras clave: horticultura, pequeña producción, áreas periurbanas

\begin{abstract}
The production of vegetables is essential to sustain the national supply of fresh food. The main production area in Chile is the Santiago Metropolitan Region (RMS). The objective of this research is to characterize the vegetable growers of the RMS, as well as to determine their attitudes towards their activity. For this, a total of 170 producers distributed in 12 communes of the region were surveyed in person. The data obtained was treated by descriptive techniques. The most prominent of the results are the following: I) the average age is 54.14 years, II) $54.5 \%$ of the respondents have no formal education or have completed only primary education, III) there is good access to services and mobilization, IV) the productive hectares average is 7.68 , but with high dispersion, V) the use of greenhouses, organic farming and hydroponics is very limited; as well as the hiring of permanent workers, VI) the use of ICT - except cellular phone - is scarce, VII) the declared innovation focuses on technical-productive aspects, VIII) most of the respondents do not associate, their relationship with the public sector is limited, they sell through intermediaries and do not keep records of their operations. Regarding their attitudes, they evaluate positively their work; however there is uncertainty about the future. We can conclude that it is important to carry out actions that result in an improvement not only of the technical-productive aspects, but also those related to management, marketing and access to ICT. That must be accompanied by an exchange with the farmers that allow them to be convinced of the opportunities to incorporate certain modifications in their work.
\end{abstract}

Keywords: vegetable cultivation, small-scale farming, periurban areas

1 Departamento de Economía Agraria y Centro Cultiva UChile, Facultad de Ciencias Agronómicas, Universidad de Chile. Santa Rosa 11315, La Pintana, Santiago, Chile.

2 Departamento de Agroindustria y Enología y Centro Cultiva UChile, Facultad de Ciencias Agronómicas, Universidad de Chile. Santa Rosa 11315, La Pintana, Santiago, Chile.

* Autor por correspondencia: sofiaboza@u.uchile.cl.

Fecha de Recepción: 22 junio, 2018.

Fecha de Aceptación: 22 septiembre, 2018.

DOI: 


\section{Introducción}

Conforme a la Encuesta de Superficie Hortícola del Instituto Nacional de Estadísticas, en Chile el cultivo de hortalizas alcanzó en 2017 las 70.706 hectáreas. La Región Metropolitana de Santiago (RMS) concentra más del $30 \%$ de dicha superficie.

La relevancia en la horticultura nacional de la RMS se da a pesar de su marcado carácter urbano. En la RMS habita el 40,5\% de la población chilena según cifras del último censo poblacional (INE, 2017). Se encuentra dividida en 52 comunas, de las cuales solamente 18 son rurales. En efecto, el continuado crecimiento del Gran Santiago ha conllevado una muy significativa merma de los suelos agrícolas regionales a través de los años, concentrándose actualmente la actividad en zonas clasificables como periurbanas (Zamora et al., 2012). La competencia con la ciudad por recursos como suelo o mano de obra es de hecho característica de los periurbanos, considerados "territorios limítrofes" (Barsky, 2005).

Como en otros cultivos intensivos en mano de obra, la agricultura familiar (AF) tiene una participación muy relevante en la producción hortícola nacional (Berdegué \& Rojas, 2014), situación que se replica en el caso de la RMS. A nivel general, la AF en Chile se caracteriza por un alto promedio de edad, limitado acceso a tecnologías, escaso uso de herramientas de gestión, canales de comercialización poco desarrollados y muy baja adhesión a asociaciones (Boza et al., 2015). En consecuencia, podemos inferir que parte importante de los horticultores presentes en la RMS arrastran falencias propias de la pequeña producción, junto con la ubicación en un territorio con continuas presiones para el cambio hacia actividades no agrícolas. Todo ello pondría en peligro su sustentabilidad en el tiempo.

Sin embargo, la cercanía con el Gran Santiago también puede suponer una ventaja para la horticultura regional, dado que es el principal mercado a nivel nacional. Así mismo, para la Seguridad Alimentaria y Nutricional (SAN) en la RMS, la oferta de productos hortícolas frescos desde las áreas periurbanas es clave. En Chile, según cifras recientes, más de un tercio de la población es obesa, casi el $40 \%$ padece de sobrepeso y solo el 5\% se alimenta de forma saludable (Minsal, 2017; Universidad de Chile, 2014). Un estudio para la RMS evidencia que, en efecto, el acceso a hortalizas frescas depende de las características del ambiente alimentario; y entre otros factores, de la presencia y diversidad de oferta de productos, así como de la seguridad en el abastecimiento (Espacio y Fomento, 2016).
El objetivo de la presente investigación es precisamente caracterizar a los agentes que constituyen el eslabón inicial de la oferta de hortalizas procedente de la RMS, esto es, a los productores. Así mismo, se busca identificar sus opiniones personales respecto a su actividad. Consideramos que este esfuerzo constituye un aporte para el diseño de políticas públicas que contribuyan al fomento del rubro y a la mejora de la SAN regional y nacional, ya que facilita información actualizada desde un enfoque (datos micro y con presencia de aspectos tanto objetivos como subjetivos) escasamente explorado.

\section{Materiales y métodos}

La información analizada en el presente artículo se obtuvo a partir de la realización de 170 encuestas a productores hortícolas ubicados en la Región Metropolitana. Los agricultores encuestados se distribuyen en las comunas de Calera de Tango, Colina, Curacaví, El Monte, Isla de Maipo, Lampa, María Pinto, Melipilla, Paine, Pirque, San Bernardo y Talagante. La muestra total fue estimada por conveniencia, así como la identificación de los individuos.

Las encuestas fueron realizadas de manera presencial durante los meses de enero y febrero de 2017. Con posterioridad se llevó a cabo un proceso de validación y codificación de la información levantada. Las preguntas que se consideraron en la encuesta se dividieron en las siguientes categorías: I) identificación de los encuestados, II) perfil socio-demográfico, III) caracterización técnico-productiva de la explotación hortícola, IV) acceso a TIC, realización de innovaciones y certificaciones, V) mecanismos de gestión predial y de comercialización de la producción, y VI) actitudes acerca de su actividad.

En el cuestionario se utilizaron preguntas cerradas de elección única - tanto dicotómica como politómica - para los ítems del I) al V). Para la última categoría se recurrió a una escala Likert de cinco niveles, en este caso, 1: "totalmente en desacuerdo", 2: "en desacuerdo", 3: "indiferente", 4: "de acuerdo", y 5: "totalmente de acuerdo". La información obtenida fue tratada mediante técnicas de análisis descriptivo.

\section{Resultados y discusión}

\section{Antecedentes generales de los encuestados}

En primer lugar, se consultó a los encuestados acerca de sus características generales: género, 
edad y nivel educacional más alto completado. Los resultados obtenidos pueden observarse en la Tabla 1.

\begin{tabular}{lrc}
\multicolumn{3}{c}{$\begin{array}{c}\text { Tabla 1. Antecedentes generales de los } \\
\text { horticultores encuestados }\end{array}$} \\
\hline Categoría & Frecuencia & Porcentaje \\
\hline Género & 151 & 88,82 \\
Hombre & 19 & 11,18 \\
Mujer & & \\
Edad & 0 & 0 \\
Menos de 25 años & 20 & 11,76 \\
Entre 25 y 39 años & 59 & 34,71 \\
Entre 40 y 54 años & 69 & 40,59 \\
Entre 55 y 69 años & 22 & 12,94 \\
Más de 70 años & & \\
Nivel de educación & 28 & 16,77 \\
Ninguno & 63 & 37,72 \\
Básica & 48 & 28,74 \\
Secundaria & 18 & 10,78 \\
Técnica & 10 & 5,99 \\
Superior & &
\end{tabular}

El 88,82\% de los encuestados fueron hombres y solo el 11,18\% mujeres. Estas cifras están notoriamente por debajo del $30 \%$ de jefatura predial femenina que se estimaba en Chile ya en 2007 (Qualitas, 2010). La composición de género tan mayoritariamente masculina hace esperable una menor incidencia de ciertas situaciones que son manifiestas en el caso de las mujeres jefas prediales, sobre todo en la pequeña agricultura. Por ejemplo, la necesidad de compatibilizar la producción con la vida familiar o la visión de la actividad agrícola como fuente complementaria de ingresos, más que como un negocio en sí mismo (Galié et al., 2013). De hecho, existe evidencia para el caso de la zona central de Chile de la continua tensión para la mujer rural entre los roles reproductivo y doméstico, que deriva en una menor inserción productiva y laboral cuando viven en pareja (Fawaz \& Rodríguez, 2013).

El promedio de edad de los encuestados fue de 54,14 años, con más de la mitad por encima de los 55 años. La continuada migración de los jóvenes desde zonas rurales a urbanas puede ser parte importante de la explicación. Según cifras del Banco Mundial la población rural chilena ha decrecido en un $0,8 \%$ anual en las últimas dos décadas, mientras que la urbana ha aumentado en un 1,5\%. Esta situación es coincidente en el caso de la zona central de Chile con las investigaciones de Castro (2012) y Valdés y Rebolledo (2015).
Respecto al nivel educacional de los encuestados, un 54,5\% de ellos no tiene educación formal completa o ha finalizado solo la educación básica. Estas cifras son notoriamente más altas que el promedio nacional recogido en la encuesta CASEN para 2015, del 29,3\% de personas mayores de 25 años en las mismas circunstancias; sin embargo, resultan más cercanas a lo que sucede en zonas rurales. Del mismo modo, para el caso de la educación superior se observa una amplia brecha respecto al promedio nacional que es del 19,8\%.

\section{Perfil socio-demográfico}

En la segunda parte de la encuesta se consultó a los productores acerca de las características básicas de su hogar, del territorio donde habitan y de su vivienda, concretamente la composición del hogar, acceso a servicios básicos y movilización.

El número de miembros que componen el hogar del encuestado - además de ellos mismos es en promedio de 2,46 personas. Un $58,13 \%$ de los productores tiene 2 o 3 miembros en su hogar y solamente un 17,9\% tiene 4 o más. Estos resultados son cercanos a los del Censo 2017, donde se estima que los hogares chilenos tienen un promedio de 3,1 miembros. En un 68,6\% de los casos, ninguno de los miembros del grupo familiar era menor de 18 años, lo que podría deberse al elevado promedio de edad de los encuestados. La mayor parte de los agricultores, un $69,76 \%$, vive en pareja.

En relación con los servicios básicos a los cuales tienen acceso en el territorio donde se encuentra su vivienda, el 94,12\% de los encuestados señaló la disponibilidad de atención de salud, siendo el consultorio la opción mencionada como de uso más habitual en el 60,87\% de los casos. Por su parte, el $94,12 \%$ de los encuestados tiene acceso a servicios de educación. Respecto al nivel más alto que se cursa en dichas entidades, tenemos en este orden: secundaria (69 individuos), superior (51 individuos), técnica (39 individuos) y básica (3 individuos). Por otro lado, el 85,88\% de los encuestados aseguró tener agua potable en su domicilio, aunque solo el $22,35 \%$ cuenta con alcantarillado. Un 99,41\% de los encuestados tiene electricidad en su vivienda, un 98,24\% telefonía fija, pero solo un 51,76\% conexión a Internet. El buen acceso a servicios puede deberse a la ubicación periurbana de los encuestados, ya que es coincidente con otros estudios anteriores para pequeños productores agrícolas ubicados en la RMS (Boza et al., 2016). 
Respecto a su movilización, el $81,07 \%$ de los encuestados dijo utilizar preferentemente locomoción particular. El bus rural es la segunda opción señalada con un 11,24\%. Cuando se consultó a los productores sobre el estado en el que consideran que se encuentra la red vial que acostumbran utilizar, las opiniones estuvieron divididas, aunque fueron mayoritariamente positivas. Estos resultados son muy relevantes, dado que es esperable que la autonomía para movilizarse les facilite a los agricultores el acceso a mercados tanto para sus productos como para la adquisición de insumos, al igual que la realización de trámites.

\section{Caracterización técnico-productiva de la explotación}

A continuación, los productores fueron consultados respecto al perfil técnico-productivo de sus explotaciones. De manera específica se les preguntó sobre tamaño, régimen de tenencia, especies hortícolas cultivadas y modos de producción, así como ingresos por venta y mano de obra requerida.

Las explotaciones que manejan los encuestados tienen en promedio 10,57 hectáreas totales y 7,68 hectáreas productivas. Sin embargo, un 51,76\% de los encuestados cuenta con menos de 5 hectáreas totales y un $65,88 \%$ productivas. Según cifras del último Censo Agropecuario (INE, 2007), los horticultores de la RMS tienen un promedio de 6,14 hectáreas en producción. El régimen de tenencia es arriendo para el $50,6 \%$ de los productores y propiedad para el $44,7 \%$. Los productos con mayor superficie cultivada son papa, cebolla, choclo y poroto, lo que en términos generales es coincidente con las cifras a nivel regional.

Casi el total de la superficie se cultiva al aire libre y el uso de invernaderos es muy limitado. El riego se realiza principalmente por surco. La gran mayoría de los agricultores usa técnicas convencionales; el cultivo orgánico e hidropónico es minoritario. En todo caso, la presencia de la agricultura orgánica en la horticultura nacional es en general baja: el total nacional registrado en 2016 era de solo 500 hectáreas (Eguillor, 2017). Respecto a la hidroponía, los resultados obtenidos son más sorprendentes, ya que es frecuente en la horticultura de la RMS. Esta diferencia se puede deber a la distribución según género de la muestra, ya que la hidroponía es especialmente común para mujeres agricultoras con muy baja disponibilidad de superficie (FAO, 2002; Boza et al., 2015).

El ingreso familiar promedio declarado por los productores fue de 952.719 pesos mensuales, aunque con importantes divergencias entre los encuestados. El $25 \%$ de los productores de menor ingreso estaba por debajo de los 400.000 pesos mensuales y el 50\% de los 525.000 pesos. La mayor parte de los encuestados, $77,97 \%$, dijo no trabajar fuera del predio. El valor de las ventas mensuales por explotación asciende a 2.412.975 pesos. La brecha entre productores es también alta en este caso, lo que a priori podría explicarse por las diferencias de tamaño entre los predios. Sin embargo, cuando consideramos el valor de las ventas por hectárea, también hay notables divergencias. En este caso son 14.196 .693 pesos anuales; sin embargo, el $50 \%$ de los productores están por debajo de los 3.500 .000 pesos. La productividad, así como probablemente el rubro específico y modelo de comercialización utilizado, tienen posiblemente un impacto en los ingresos.

Respecto al número de trabajadores que tienen contratados de forma permanente, el promedio fue de 1,43. Sin embargo, el 52,94\% de los encuestados no tenía ningún trabajador permanente y un $16,47 \%$ solamente uno. Estas cifras pueden ser explicadas por una parte porque los pequeños productores, y en especial la agricultura familiar, se caracterizan por recurrir al trabajo de los miembros del hogar (Garner \& de la O, 2014). En relación con la participación de al menos un familiar en las labores prediales, se da para un $45,34 \%$ de los encuestados. Por otro lado, en las labores agrícolas priman en muchos casos las relaciones de índole temporal, así como los contratos de palabra basados en la confianza, dado que no se ve la necesidad de formalizarlos (Maloney, 2004).

\section{Acceso a TIC, innovación y certificaciones de los encuestados}

Con el fin de complementar la caracterización técnico-productiva de las explotaciones, se consultó a los encuestados acerca de la penetración de las TIC en sus negocios, las innovaciones realizadas recientemente y el uso de certificaciones. Los principales resultados se pueden observar en la Tabla 2.

En primer lugar, es notoria la baja penetración de las principales TIC entre los encuestados, excepto para la telefonía celular. Estos resultados son coherentes con investigaciones previas que señalan que existe una relación directa entre los años de escolaridad del responsable de una empresa y la inserción de las TIC en ella (Goldfarb \& Prince, 2008). En la misma línea, los encuestados fueron consultados acerca 
Tabla 2. Uso de TIC, innovación y certificaciones de los encuestados

\begin{tabular}{|c|c|c|c|c|}
\hline Categoría & Sí & $\%$ & No & $\%$ \\
\hline Uso de celular & 160 & 94,12 & 10 & 5,88 \\
\hline Uso de computador & 55 & 32,35 & 115 & 67,65 \\
\hline Uso de correo electrónico & 53 & 31,18 & 117 & 68,82 \\
\hline Realización de trámites online & 35 & 20,59 & 135 & 79,41 \\
\hline \multicolumn{5}{|c|}{ Innovación en los últimos 5 años en: } \\
\hline Especies & 91 & 53,53 & 79 & 46,47 \\
\hline Manejos de campo & 63 & 37,06 & 107 & 62,94 \\
\hline Prácticas de gestión & 19 & 11,18 & 151 & 88,82 \\
\hline Tecnologías productivas & 46 & 27,06 & 124 & 72,94 \\
\hline TIC & 8 & 4,71 & 162 & 95,29 \\
\hline Envases o etiquetado & 9 & 5,33 & 160 & 94,67 \\
\hline Procesamiento & 8 & 4,71 & 162 & 95,29 \\
\hline \multicolumn{5}{|l|}{ La explotación cuenta con: } \\
\hline Buenas Prácticas Agrícolas & 42 & 24,71 & 128 & 75,29 \\
\hline HACCP & 0 & 0 & 170 & 100 \\
\hline ISO & 2 & 1,18 & 168 & 98,82 \\
\hline Resolución sanitaria & 19 & 11,18 & 151 & 88,82 \\
\hline
\end{tabular}

del principal medio de comunicación que usan para informarse. Los resultados fueron, por orden de importancia: televisión $(54,76 \%)$, radio $(19,64 \%)$, Internet $(18,45 \%)$ y prensa escrita $(7,14 \%)$. Estos datos resultan relevantes, entre otras situaciones, a la hora de plantear una estrategia para dar a conocer a los productores los instrumentos públicos de apoyo, o de entregarles información de interés, como previsiones climáticas o de precios.

En cuanto a la innovación reciente que señalan los encuestados, se centra en el cultivo de nuevas especies y, de forma secundaria, en el manejo del campo y la inclusión de tecnologías productivas. Las innovaciones relativas a gestión, uso de TIC y agregación de valor en la post cosecha son muy marginales. Según datos del Ministerio de Economía (2018) la tasa de innovación en el sector agrícola es una de las más bajas de la economía nacional $(9,5 \%)$. Así mismo, se evidencia una brecha en la innovación según la orientación exportadora. En este caso, si bien las cifras no son tan reducidas, es posible que exista una confusión conceptual entre innovación y recepción de transferencia tecnológica. En relación con los permisos y las certificaciones, aunque las opciones presentadas son todas minoritarias, la de Buenas Prácticas Agrícolas es la más habitual. La resolución sanitaria tiene una muy baja incidencia, no obstante, solamente se requiere cuando al producto primario se le realiza alguna transformación. El escaso interés por certificarse puede deberse a que la horticultura está mayoritariamente enfocada al mercado local, el cual por ahora no es tan exigente en este sentido. De hecho, las investigaciones sobre el impacto de la adopción de certificaciones privadas por parte de la pequeña agricultura evidencian un mejor acceso a cadenas comerciales de alto valor agregado (Beghin et al., 2015).

\section{Mecanismos de gestión predial y de comercialización}

Adicionalmente, se solicitó a los productores información acerca de sus mecanismos para gestionar sus explotaciones y comercializar. Para ello se les preguntó acerca de la formalización de sus actividades, el uso de registros, el acceso a financiamiento, el apoyo asociativo, la participación en programas públicos y los canales de venta.

Un 60,59\% de los productores aseguró que su explotación tiene inicio de actividades en el Servicio de Impuestos Internos. Esta cifra es notablemente superior al promedio para el sector primario a nivel nacional, del 26,2\% conforme al Ministerio de Economía. Sin embargo, se detectan inconsistencias posteriores en las respuestas, ya que solo el $28,15 \%$ de aquellos que dijeron tener inicio de actividades fueron capaces de especificar el tipo de personalidad jurídica y el 79,61\% su régimen de tributación.

Por otro lado, el 85,29\% de los encuestados señaló que eran ellos mismos quienes se encargaban de la administración predial. Un 58,24\% de los agricultores dijo no tener registros económicos - de producción y costos - relativos a la explotación. Por otro lado, solo el 26,63\% de los encuestados afirmó pertenecer a alguna asociación técnico-productiva. El bajo nivel educacional de los productores puede estar impactando en el escaso asociativismo, dado que para el caso de Chile se ha demostrado una relación directa con los años de escolaridad del jefe predial (Jaime et al., 2010).

En el financiamiento para las inversiones y gastos operativos prediales, el $38,31 \%$ de los encuestados ha recibido subsidios y el $44,71 \%$ crédito en los últimos 5 años. La principal fuente de los créditos fue el Instituto de Desarrollo Agropecuario, INDAP, seguido del Banco Estado. La banca privada tuvo, sin embargo, una participación bastante baja. De hecho, el nivel de bancarización de los productores es relativamente débil: solo un 46,47\% de los encuestados aseguró utilizar cuenta para administrar los flujos monetarios 
de la explotación. Los efectos del acceso a crédito en la productividad - y en consecuencia en los ingresos prediales - para el caso específico de Chile, se evidencian heterogéneos, dependiendo del rubro e incluso del capital social del productor (von CramonTaubadel \& Saldías, 2014; Reyes \& Lensink, 2011).

El 48,24\% de los encuestados dijo haber sido beneficiario de al menos un programa público en los últimos 5 años. De ellos el 69,51\% perteneció al Programa de Desarrollo Local, PRODESAL. Cada productor ha sido 7,32 años en promedio beneficiario del programa señalado. PRODESAL es el programa público más importante de extensión agrícola en términos de cobertura y número de beneficiarios en Chile. Así mismo, PRODESAL se caracteriza, además de su carácter comprehensivo, por no tener criterios de egreso definidos, lo que posibilita participaciones de largo plazo (Namdar-Irani \& Sotomayor, 2011). Solamente un $11,18 \%$ de los encuestados afirmó haber recibido apoyo del sector privado en los últimos 5 años.

Por último, en relación con la comercialización, los intermediarios y mayoristas son el principal canal para más de la mitad de los encuestados. De manera secundaria aparece la venta directa en predio y en ferias. La exportación y los supermercados son muy minoritarios. Estos resultados son coincidentes con la realidad nacional. La pequeña agricultura chilena, sobre todo en el caso de hortofrutícolas, canaliza sus ventas en gran medida a través de la cadena productor-intermediario-centrales de abastecimientoferias libres y, en menor proporción, de forma directa (Rivas, 2012).

\section{Actitudes acerca de su actividad}

Por último, se presentó a los encuestados una serie de afirmaciones referentes a su actividad como productores hortícolas para que pudieran valorar su grado de acuerdo con ellas en una escala de 1 ("totalmente en desacuerdo") a 5 ("totalmente de acuerdo"). Los principales resultados se muestran en la Tabla 3.

Por un lado, se evidencia una elevada confianza de los agricultores en la calidad de sus productos, dada la alta valoración de las afirmaciones: "Considero que mis hortalizas son inocuas" (promedio 4,88) y "Considero que mis hortalizas son de buena calidad" $(4,74)$. En lo relativo a las técnicas productivas utilizadas, por lo general existe acuerdo acerca de su conveniencia, siendo valoradas en este orden: propagación de material vegetal $(4,20)$; fertilización de suelo $(4,07)$; manejo de residuos $(3,84)$; control de malezas $(3,83)$; control de plagas y enfermedades $(3,79)$ y riego $(3,79)$. Así mismo, hay satisfacción con el proceso de venta de las hortalizas: "La forma de pago por la venta de mis productos es de mi agrado" $(4,70)$ y "La venta de mis hortalizas se realiza en un momento oportuno" $(4,48)$. Pero no es igual en lo relativo al precio obtenido: "El precio de venta de mis hortalizas es bueno" $(3,02)$. A pesar de que en los hechos no se vea tan reflejado, existe alto grado de acuerdo en la utilidad de la formalización, la innovación (especialmente productiva) y la pertenencia a asociaciones. Respecto al apoyo del sector público, hay acuerdo respecto a su impacto positivo: "Los instrumentos públicos de apoyo que he recibido han sido útiles para mi actividad" $(4,33)$. No obstante, se considera que la información disponible, los procedimientos de acceso y la magnitud de dichos apoyos no son los más adecuados: "Los instrumentos de apoyo disponibles para el sector hortícola son suficientes" $(2,41)$; "Considero que la información disponible sobre instrumentos de apoyo es suficiente" $(2,42)$; "La postulación a instrumentos de apoyo para el sector hortícola es sencilla" $(2,89)$.

Respecto a la visión general acerca de su actividad, los resultados sugieren una cierta incertidumbre en torno a su futuro: "Tengo una visión positiva sobre el futuro de mi actividad hortícola" $(3,44)$. Un punto crítico para poder crecer es la disponibilidad de recursos financieros: "Necesito mayor inversión para hacer crecer mi producción" $(4,65)$. No existe un claro acuerdo en lo referente a la contribución que los ingresos generados por la horticultura signifiquen para el hogar, así como en el tiempo dedicado a la actividad: "Considero que los ingresos que genera mi actividad permiten que mi grupo familiar tenga acceso a una buena calidad de vida" $(3,68)$ y "Considero que la cantidad de tiempo que trabajo en mi predio hortícola es adecuada" $(3,67)$. Por el contrario, es compartida la percepción de que se realiza una actividad sustentable desde el punto de vista ambiental: "Considero que mi actividad es amigable con el medio ambiente" $(4,36)$.

Por último, la disposición a pagar por asesorías especializadas es baja, sobre todo para aquellas relativas a aspectos de gestión y comerciales. En cuanto a las asesorías técnico-productivas, ello puede deberse a que, principalmente en el caso de los pequeños productores, estos acostumbran a recibir ese tipo de beneficios de manera gratuita dentro de programas como PRODESAL. Para las asesorías 
Tabla 3. Valoración de afirmaciones relativas a actitudes de los encuestados frente a su actividad hortícola

\begin{tabular}{|c|c|c|}
\hline Afirmación & Media & D.E. \\
\hline 1. Necesito mayor inversión para hacer crecer mi producción & 4,65 & 0,83 \\
\hline 2. Considero que mis técnicas de riego son adecuadas & 3,79 & 1,11 \\
\hline 3. Considero que mis técnicas de fertilización de suelo son adecuadas & 4,07 & 0,92 \\
\hline 4. Considero que mis técnicas de propagación de material vegetal son adecuadas & 4,20 & 0,77 \\
\hline 5. Considero que mis técnicas de control de malezas son adecuadas & 3,83 & 0,98 \\
\hline 6. Considero que mis técnicas de control de plagas y enfermedades son adecuadas & 3,79 & 1,06 \\
\hline 7. Considero que mis técnicas de manejo de residuos son adecuadas & 3,84 & 1,02 \\
\hline 8. Considero que mis hortalizas son de buena calidad & 4,74 & 0,50 \\
\hline 9. Considero que mis hortalizas son inocuas & 4,88 & 0,36 \\
\hline 10. Estoy dispuesto a pagar por asesorías especializadas sobre técnicas de producción & 3,40 & 1,34 \\
\hline 11. La venta de mis hortalizas se realiza en un momento oportuno & 4,48 & 0,81 \\
\hline 12. La forma de pago por la venta de mis productos es de mi agrado & 4,70 & 0,67 \\
\hline 13. El precio de venta de mis hortalizas es bueno & 3,02 & 1,13 \\
\hline 14. Estoy dispuesto a pagar por asesorías especializadas sobre comercialización & 2,90 & 1,33 \\
\hline 15. Considero que la formalización es importante para el éxito de mi actividad & 4,50 & 0,98 \\
\hline 16. Considero que los trámites para formalizarse son sencillos & 3,34 & 1,11 \\
\hline 17. Considero que los trámites para obtener certificaciones son sencillos & 2,64 & 0,90 \\
\hline 18. Estoy dispuesto a pagar por asesorías especializadas sobre gestión & 2,76 & 1,25 \\
\hline 19. Tengo una visión positiva sobre el futuro de mi actividad hortícola & 3,44 & 1,34 \\
\hline 20. La introducción de innovaciones en mi sistema productivo ha sido beneficiosa & 4,32 & 0,96 \\
\hline 21. La introducción de innovaciones en mi sistema comercial ha sido beneficiosa & 3,90 & 1,04 \\
\hline 22. La introducción de innovaciones en mi sistema de gestión ha sido beneficiosa & 3,96 & 0,96 \\
\hline $\begin{array}{l}\text { 23. Considero que los ingresos que genera mi actividad permiten que mi grupo familiar tenga acceso } \\
\text { a una buena calidad de vida }\end{array}$ & 3,68 & 1,16 \\
\hline 24. Considero que la cantidad de tiempo que trabajo en mi predio hortícola es adecuada & 3,67 & 1,24 \\
\hline 25. Considero que mi actividad es amigable con el medio ambiente & 4,36 & 0,79 \\
\hline 26. Los instrumentos de apoyo disponibles para el sector hortícola son suficientes & 2,41 & 0,93 \\
\hline 27. Los instrumentos públicos de apoyo que he recibido han sido útiles para mi actividad & 4,33 & 0,91 \\
\hline 28. Los instrumentos privados de apoyo que he recibido han sido útiles para mi actividad & 3,74 & 1,02 \\
\hline 29. Considero que la información disponible sobre instrumentos de apoyo es suficiente & 2,42 & 1,09 \\
\hline 30. La postulación a instrumentos de apoyo para el sector hortícola es sencilla & 2,89 & 1,10 \\
\hline $\begin{array}{l}\text { 31. Considero que la existencia de asociaciones técnico-productivas o comerciales son beneficiosas } \\
\text { para quienes las conforman }\end{array}$ & 4,23 & 0,99 \\
\hline
\end{tabular}

en gestión y comercialización, es probable que exista desconocimiento de los beneficios que pueden suponer.

Aunque el análisis de actitudes es más frecuente en el caso de los estudios del consumidor, se ha aplicado previamente para identificar las percepciones de los agricultores en el caso de Chile (ej. Boza et al.,2016; Mora et al., 2013). En términos generales, estas investigaciones muestran que, incluso entre pequeños agricultores, existen distintas maneras de percibir su actividad, sobre todo en relación con la actitud frente al cambio. Lo anterior a su vez está condicionado por las características propias de cada productor, como por ejemplo la edad, el nivel educacional, el género o el desarrollo tecnológico.

\section{Conclusiones}

El presente artículo permite concluir acerca de algunas características generales de la población bajo estudio. En primer lugar, la edad de los agricultores es relativamente elevada, ya que la mayor parte $(53,5 \%)$ está por encima de los 55 años y un grupo muy minoritario $(11,76 \%)$ por debajo de los 40 . Otro resultado relevante es el bajo nivel educacional de los productores, con más de la mitad $(54,5 \%)$ con solo básica, concluida o inconclusa. Lo anterior, es de esperar que condicione la transmisión de conocimientos y que incida en la voluntad de introducir innovaciones en su quehacer. 
Respecto al grupo familiar, el promedio de integrantes es de 3,46. En la mayor parte de los casos $(68,6 \%)$ ninguno de ellos es un menor. El perfil etario de los productores muy probablemente tiene incidencia en la conformación de sus hogares en este sentido. Un $45,34 \%$ de los productores tiene otro miembro del grupo familiar que trabaja en el predio. La gran mayoría de los agricultores tiene acceso a servicios básicos y a movilización particular. En esto, el hecho de estar ubicados precisamente en la RMS puede estar impactando.

En relación con los sistemas productivos, la superficie utilizada por los encuestados para el cultivo de hortalizas es en promedio 7,68 hectáreas. No obstante, la dispersión de los datos es bastante amplia y la mayor parte de los agricultores $(65,88 \%)$ está por debajo de las cinco hectáreas. Los productores son principalmente dueños $(44,7 \%)$ o arrendatarios $(50,6 \%)$ de sus predios. Los cultivos más frecuentes son papa, cebolla, poroto y choclo. La mayor parte $(52,94 \%)$ no tiene ningún trabajador permanente y en un $85,29 \%$ se encargan ellos mismos de la administración predial. Casi la totalidad de la superficie se cultiva conforme a métodos convencionales, y la hidroponía y las técnicas orgánicas son muy minoritarias, al igual que las certificaciones. La alta incidencia de la comercialización a través de intermediarios puede estar relacionada con estas cifras.

El uso de TIC con fines relacionados con la explotación es también muy escaso, excepto en lo que respecta al celular. Las innovaciones, cuando existen, se circunscriben a lo técnico-productivo. La participación en programas públicos está muy focalizada en PRODESAL y en el crédito de INDAP. Respecto a lo último, el acceso a otro tipo de financiamiento es muy escaso. La mayor parte de los encuestados $(58,24 \%)$ no mantiene registros económicos de su actividad y una minoría $(26,63 \%)$ participa de algún tipo de asociación. Respecto a sus actitudes, destaca la valoración positiva de la manera en la cual realizan su actividad productiva; sin embargo, existe incertidumbre de cara al futuro de esta.

A partir de los resultados obtenidos se puede recomendar llevar a cabo acciones que deriven en una mejora no sólo de los aspectos técnico-productivos, sino también de aquellos relacionados con la gestión, la comercialización y el acceso a TIC. Lo anterior debe ir acompañado de un intercambio con los productores que les permita convencerse de las oportunidades de incorporar ciertas modificaciones en su quehacer.

\section{Agradecimientos}

Esta investigación fue desarrollada dentro del proyecto Centro de Extensión Hortícola financiado por el concurso de INNOVA-CORFO Centro de Extensionismo Tecnológico, línea Alimentos Saludables. Los autores agradecen asimismo a los profesionales que colaboraron en el levantamiento de la información.

\section{Literatura citada}

Barsky, A.

2005. El periurbano productivo, un espacio en constante transformación. Introducción al estado del debate, con referencias al caso de Buenos Aires. Scripta Nova, 9 (194).

Beghin, J.; Maertens, M.; Swinnen, J.

2015. Non-Tariff Measures and Standards in Trade and Global Value Chains. Bioeconomics Working Paper Series, 2015/02.

Berdegué, J.; Rojas, F.

2014. La agricultura familiar en Chile. Rimisp Grupo de Trabajo Desarrollo con Cohesión Territorial. Documento de Trabajo $\mathrm{N}^{\circ} 152$.

Boza, S.; Cortés, M.; Guzmán, F.

2015. Caracterización de pequeños empresarios agrícolas beneficiarios de programas de desarrollo local en la Región Metropolitana, Chile. Revista IDESIA, 33(1): 135-142.

Boza, S.; Cortés, M.; Muñoz, T. 2016. Estrategias de desarrollo rural con enfoque de género en Chile: el caso del programa Formación y Capacitación para Mujeres Campesinas. Revista Civilizar, 16 (30): 63-75.
Castro, A.

2012. Familias Rurales y sus Procesos de Transformación: Estudio de Casos en un Escenario de Ruralidad en Tensión. Psicoperspectivas, 11(1): 180-203.

Eguillor, P.

2017. Agricultura orgánica: agosto de 2017. Santiago, Chile: ODEPA. 24 p.

Espacio y Fomento

2016. Estudio para el desarrollo de los mercados locales para mejorar el acceso a frutas y hortalizas frescas en las regiones Metropolitana y del General Libertador Bernardo FAO O'Higgins. ODEPA. Santiago, Chile. 46 p.

2002. Agricultura Urbana en la Región Metropolitana de Santiago de Chile: Situación de las Empresas Familiares Hidropónicas - Estudio de casos. FAO. Santiago, Chile. 31 p. Fawaz, J.; Rodríguez, C.

2013. Mujeres rurales y trabajo en Chile central. Actitudes, factores y significaciones. Cuadernos de Desarrollo Rural, 10 (72): 47-68. 
Galiè, A.; Jiggins, J.; Struik, P. C.

2013. Women's identity as farmers: A case study from ten households in Syria. Wageningen Journal of Life Sciences, 64-65: 25-33.

Garner, E.; De la O, A. P.

2014. Identifying the "family farming": an informal discussion of the concepts and definitions. ESA-FAO Working Paper, No. 14-10.

Goldfarb, A.; Prince, J.

2008. Internet adoption and usage patterns are different: Implications for the digital divide. Information Economics and Policy, 20 (1): 2-15.

Jaime, M.; Salazar, C.; Araya, S.; Vergara, J.

2010. Niveles de participación en la pequeña agricultura ¿De qué depende que los productores se involucren en sus organizaciones? Economía Agraria, 14: 9-21.

Maloney, W.F.

2004. Informality revisited. World Development, 32 (7): 1159-1178.

MINSAL.

2017. Encuesta Nacional de Salud ENS Chile 2016-2017: Primeros resultados. MINSAL. Santiago, Chile. 61 p.

Mora M.; Cortés M.; Sanhueza C.; Sáenz C.

2013. Innovation requirements for the development of cactus pear for export: A new item to be incorporated to the Chilean fruit export sector. Acta Hortulturae, 995: 229-236.
Namdar-Irani, M.; Sotomayor, O.

2011. Le conseil agricole au Chili face à la diversité des agriculteurs. Cahiers Agricultures, 20: 352-358.

Qualitas

2010. Estudio de caracterización de la pequeña agricultura a partir del VII Censo Nacional Agropecuario y Forestal. Qualitas/Indap. Santiago, Chile. 37 p.

Reyes, A.; Lensink, R.

2011. The Credit Constraints of Market-Oriented Farmers in Chile. Journal of Development Studies, 47 (12): 1851-1868. Rivas, $\mathrm{T}$.

2012. Comercialización de productos hortofrutícolas en la pequeña agricultura. ODEPA. Santiago, Chile. 9 p.

Universidad de Chile.

2014. Encuesta Nacional de Consumo Alimentario. UChile. Santiago, Chile. 329 p.

Valdés, X.; Rebolledo, L.

2015. Géneros, generaciones y lugares: cambios en el medio rural de Chile Central. Polis (Santiago), 14 (42): 491-513. Von Cramon-Taubadel, S.; Saldías, R.

2014. Access to Credit and Determinants of Technical Inefficiency of Specialized Samllholder Farmers in Chile. Chilean Journal of Agricultural Research, 74 (4): 413-420.

Zamora, G.; Álvarez, L.; Gajardo, G.; Rodríguez, R.; Salinas, C. 2012. Estudio de Impacto de la Expansión Urbana Sobre el Sector Agrícola en la Región Metropolitana de Santiago. ODEPA. Santiago, Chile. 57 p. 